\title{
Short-term Prediction of Building Energy Consumption Based on GALM Neural Network
}

\author{
ZHONG Bocheng, LU Kuo, LV Dinghao, LUO Jing, FANG Xuan \\ College of Electronic \& Electrical Engineering, Shanghai University of Engineering Science, \\ Shanghai, 201620, China \\ email: bczhong@163.com
}

\begin{abstract}
Keywords: Building Energy Consumption; Short-term Prediction; ANN; Genetic Algorithm; Levenberg-Marquardt Algorithm
\end{abstract}

\begin{abstract}
In order to improve the conventional method of predicting building energy consumption using artificial neural networks (ANN), we proposed a novel neural network model to forecast building energy consumption. The model optimized the neural network based on genetic algorithm and Levenberg- Marquardt algorithm Firstly genetic algorithm was used to optimize the weight and threshold of ANN, Secondly Levenberg-Marquardt algorithm (LM) was adopted to optimize the neural network training. Then the predicting model based on the new algorithm was set up in terms of the main factors effecting the energy consumption. Furthermore, a public building electric consumption data for one month is collected to train and test the model. The testing results show that the model is more accurate and efficient than the conventional method in predicting short-term energy consumption.
\end{abstract}

\section{Introduction}

As to a particular architecture, there are many factors with the relevant of its energy consumption, such as character activities, climate, equipment operation, envelop structure and so on. Besides, they are non linear correlation with each other [1]. Artificial neural networks are parallel computational models, comprised of densely interconnected adaptive processing units, which are able to solve a wide variety of problems. Therefore, many researchers applied ANN to make building energy consumption [2]. For example, Alberto et al. [3] used two models respectively based on ANN and EnergyPlus, and they put out that both models were suitable for energy consumption forecast. Azadeh et al. [4] predicted the long-term annual electricity consumption in energy intensive manufacturing industries, and showed that the neural network is very applicable to this problem when energy consumption shows high fluctuation. Betul et al. [5] selected transparency, orientation, insulation and thickness as the input of a back propagation neural network to predict the heating energy consumption, the paper introduced how they get the samples in detail. However, conventional ANN has shortcomings of slow convergence rate and getting easy to fall into local minimum. To overcome them, Pai et al. [6] used adaptive neuro fuzzy inference system which was improved ANN by fuzzy logic to forecast suspended solids and chemical oxygen demand in the effluent from a hospital wastewater treatment plant, and increased the prediction performance. Laurent and Fariborz [7] presented the GAINN approach that used a simulation-based ANN to characterize building behavior, and then combined this ANN with a multi-objective Genetic Algorithm for optimization. To test and verify proposed method, they apply it to the optimization of thermal comfort and energy consumption in a residential house, the results showed the total simulation time was considerably reduced. Wang [8] et al. combined the global search ability of genetic algorithm (GA) with the local search ability of the BP algorithm, using GA to optimize the initial weights of the neural networks and BP algorithm to get the optimal solution in feasible region area, finally they proved the optimized model could not make faster prediction, the result was of high precision by using this new method. However, it made calculation quantity increased since adopting GA. In [9], with the same prediction target, training steps of genetic neural networks is 10 times of the LM neural networks, it indicated genetic neural networks were not 
suitable for short-term prediction. Therefore, in this paper, we proposed applying the LM algorithm to genetic neural networks to make further improvement which called GALM neural networks and set up a short-term prediction model of building energy consumption.

\section{Prediction Model Based on the GALM Neural Networks}

The algorithm we put combines the global search ability of genetic algorithm with the local search ability of the LM algorithm since the convergence rate of LM algorithm than much faster than that of BP, it will make up the extra learning time caused by GA. The learning process can be divided into two main stages,1)genetic algorithm locates the approximate solution area, 2) take this as the starting point, LM algorithm searches local area of the point, until converges to the optimal solution.

There are many factors affecting building energy consumption, take a particular architecture into consideration, they can be ranged into character activities, climate, equipment operation, and envelop structure. However, in the short run, staff activities is relatively fixed; equipment operation logs change little; envelop structure is basically unchanged. Therefore we mainly discuss the outdoor climate factors.

According to the short-term energy consumption characteristics of a certain building, we selected daily average temperature, dew-point temperature, average relative humidity and average wind-speed as the input neurons, obviously the output neuron is electric energy consumption. Therefore, the input layer has 4 neurons and the output layer has 1 .

In terms of layers, [10] has demonstrated that the three-layer BP network fully learned can approximate any nonlinear relationship with any precision, considering the complexity of prediction model, there's only 1 hidden layer. Based on the empirical formulas and repeated experimental comparison, we ultimately selected the network model in the form of 4-10-l, as shown in Fig. 1.

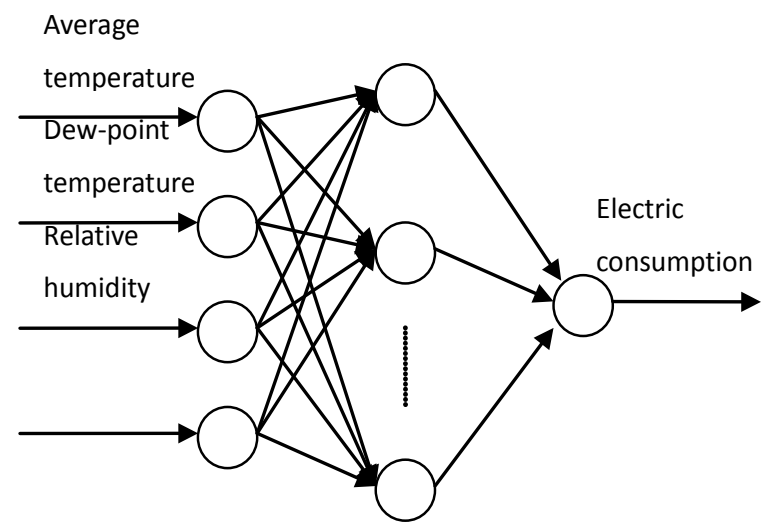

Fig.1 Prediction model structure

\section{Simulation Analysis}

The ball $R=21.6 \mathrm{~mm}, m_{b}=45 \mathrm{~g}$. To ensure the cue ball and pick and not run into low contact choose other parts of the cue for $w=16 \mathrm{~mm}$. From the law of conservation of energy, it is known that the lighter lift stick, the faster the speed at first, consider to pick cue the deformation of stress intensity factor, finally optimization for $m_{c}=34 \mathrm{~g}$. Since robot have play ball institutions, in order not to decorate with the wheel position conflict, only can pick the ball in play on electromagnetic valve electromagnet institutions. According to the electromagnet diameter and robot's height, take $L=58 \mathrm{~mm}, h=19 \mathrm{~mm}$.

The Foreign and Commonwealth Office "Main Building” make it available for public to collect a range of energy use data from their offices at King Charles Street. It's generated in real-time from data taken every 5 seconds from the on-site meters [10]. And the climate data is from 
www.weather.org. In this paper 31 days' data was gathered and divided into two parts, the first 26 days' data for training and the rest for testing. In order to improve the fault-tolerant capability of prediction model, we added noise in training sample, so 52 groups were produced for training.

Since inconsistency of the sample data, the sample should be normalized before training. Here, adopted premnmx function in MATLAB .the method is as follows:

$$
p^{\prime}=2 \frac{p-p_{\min }}{p_{\max }-p_{\min }}-1
$$

Type: $p^{\prime}$ for normalized variable; $p$ as original variable; $p_{\max }, p_{\min }$ as the maximum and minimum variables respectively. In terms of prediction model parameters:

Genetic algorithm configuration: population $n=50$, generation gen $=100$, crossover rate $P_{c}=0.5$, mutation rate $P_{m}=0.01$.

Neural networks configuration: learning rate 0.3, maximum epochs 8000, training error 0.001 . Transfer function of hidden layer is S type tangent function, and that of hidden layer is a linear function.

Comparing prediction models respectively based on genetic neural networks and GALM neural networks in the same condition, the typical training error curves of two networks are as follows selected from amount of experiments:

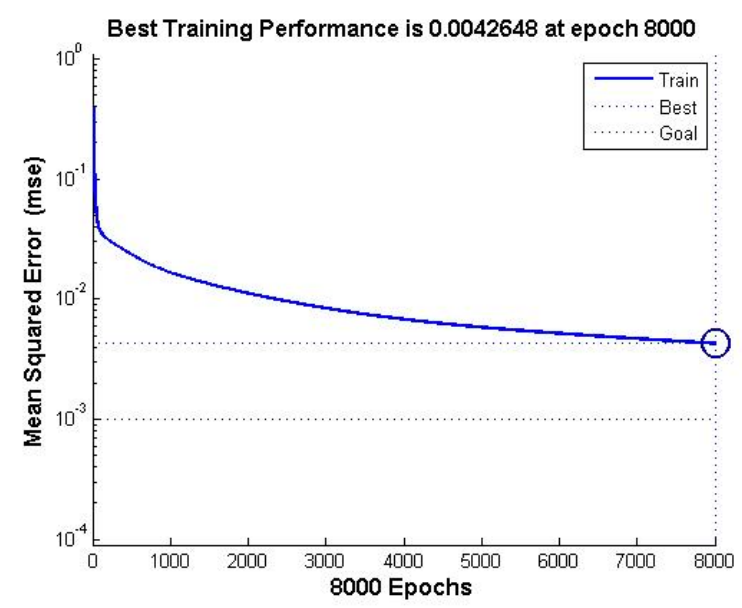

Fig.2 Training error curve of genetic neural networks

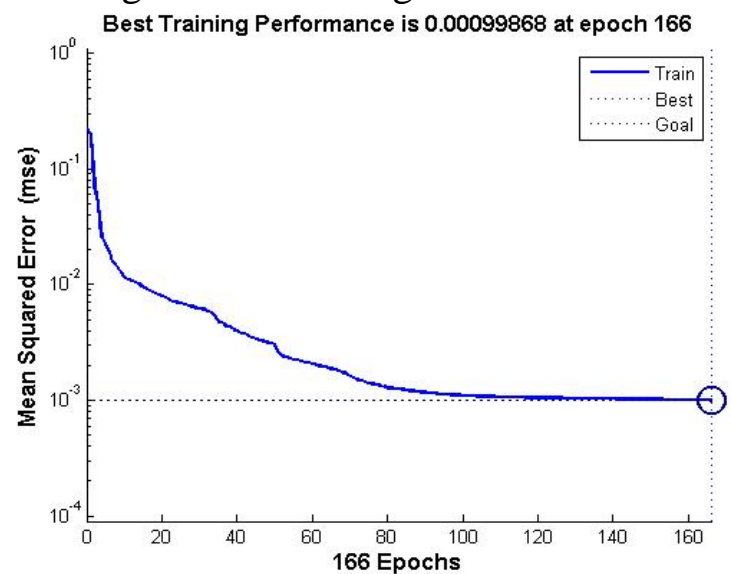

Fig.3 Training error curve of GALM

Although GA has promoted the convergence rate of BP neural networks, the bulk velocity of training process is not as fast as expected. Fig.2 shows that genetic neural networks prediction model spend a lot of time trainings, still can't reach the target even at the maximum epochs.

Likewise, experimental results show that GALM algorithm trains much faster than genetic neural networks do, achieving training goal within 200 times basically, just like Fig. 3 shown. It is because LM algorithm is second - order which compensates the additional consumed time and makes it 
meet the real-time requirements of short-term prediction.

Except for real time requirements, short-term prediction demands low error as well. Applying the trained neural networks to forecast the electric consumption in 5 days indicates that model based on GALM has high precision. After calculating we can easily get known the maximum relative error is $4.43 \%$, the average relative error is $1.06 \%$, detail shows in Tab.1.

In summary, neural networks based on GALM is suitable for making short-term prediction of building energy consumption.

Tab.1 Prediction result and error

\begin{tabular}{cccccc}
\hline Data & 27th & 28th & 29th & 30th & 31st \\
\hline True value & 14187 & 20616.5 & 20778.5 & 21040.5 & 20673 \\
Prediction value & 14815 & 20716 & 21117 & 20850 & 20815 \\
Relative error & $4.43 \%$ & $0.48 \%$ & $1.63 \%$ & $0.91 \%$ & $0.67 \%$ \\
\hline
\end{tabular}

\section{Conclusion}

According to real-time requirements in short-term building energy consumption prediction, this paper proposed GALM learning algorithm and established prediction model based on GALM neural networks. Through simulation under the same condition, the comparison results showed that GALM neural networks not only meet the real-time requirement of short-term prediction, the accuracy also satisfied the actual need engineering specifications.

\section{Acknowledgement}

In this paper, the research was sponsored by the development fund of digital logic course from Shanghai Municipal Education Commission (Project No. S201302001).

\section{References}

[1] Wong, S., Wan, K.K., Lam, T.N. Artificial neural networks for energy analysis of office buildings with daylighting.[J]. Applied Energy, 2010 87(2):551-557.

[2] Kumar, Rajesh; Aggarwal, R.K.; Sharma, J.D. Energy analysis of a building using artificial neural network: A review. [J]. Energy and Buildings 2013 65(4):352-358.

[3] Neto, Alberto Hernandez, and Flávio Augusto Sanzovo Fiorelli. Comparison between detailed model simulation and artificial neural network for forecasting building energy consumption.[J]. Energy and Buildings, 2008 40(12):2169-2176.

[4] Azadeh, A., Ghaderi, S.F., Sohrabkhani, S. Annual Electricity Consumption Forecasting By Neural Network In High Energy Consuming Industrial Sectors.[J]. Energy Conversion and Management, 2008 49(8):2272-2278.

[5] Ekici, Betul Bektas, and U. Teoman Aksoy. Prediction of building energy consumption by using artificial neural networks.[J]. Advances in Engineering Software, 2009 40(5):356-362.

[6] Pai, T.Y.,Wan, T.J., Hsu, S.T., Chang, T.C., Tsai, Y.P., Lin, C.Y., Su, H.C., Yu, L.F. Using fuzzy inference system to improve neural network for predicting hospital wastewater treatment plant effluent.[J]. Computers \& Chemical Engineering, 2009 33(7):1272-1278.

[7] Magnier, Laurent, and Fariborz Haghighat. Multiobjective optimization of building design using TRNSYS simulations, genetic algorithm, and Artificial Neural Network.[J]. Building and Environment, 2010 45(3):739-746. 
[8] CHEN Hai-ying, GUO Qiao, XU Li. 100 meters race performance prediction based on hybrid genetic neural network.[J]. Computer Simulation, 2004 21(2):89-91.

[9] Wang Weimin, Zmeureanu Radu, Rivard Hugues. Applying multi-objective genetic algorithms in green building design optimization.[J]. Building and Environment, 2005 40(11):1512-1525.

[10] Kůrková V. Kolmogorov's theorem and multilayer neural networks.[J]. Neural networks, 1992 5(3):501-506. 